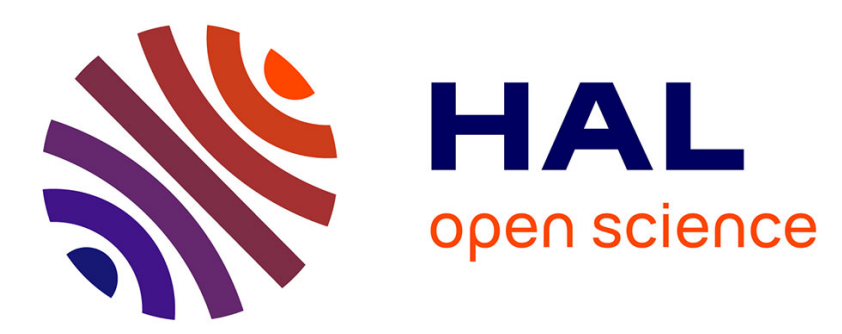

\title{
Etude de la mobilité des dislocations dans le silicium monocristallin par frottement intérieur haute température \\ P. Gadaud, J. Woirgard
}

\section{- To cite this version:}

P. Gadaud, J. Woirgard. Etude de la mobilité des dislocations dans le silicium monocristallin par frottement intérieur haute température. Revue de Physique Appliquée, 1988, 23 (5), pp.919-924. 10.1051/rphysap:01988002305091900 . jpa-00245893

\section{HAL Id: jpa-00245893 https://hal.science/jpa-00245893}

Submitted on 1 Jan 1988

HAL is a multi-disciplinary open access archive for the deposit and dissemination of scientific research documents, whether they are published or not. The documents may come from teaching and research institutions in France or abroad, or from public or private research centers.
L'archive ouverte pluridisciplinaire HAL, est destinée au dépôt et à la diffusion de documents scientifiques de niveau recherche, publiés ou non, émanant des établissements d'enseignement et de recherche français ou étrangers, des laboratoires publics ou privés. 


\title{
Etude de la mobilité des dislocations dans le silicium monocristallin par frottement intérieur haute température
}

\author{
P. Gadaud et J. Woirgard \\ Laboratoire de Mécanique et de Physique des Matériaux, E.N.S.M.A., 86034 Poitiers Cedex, France
}

(Reçu le 6 octobre 1987, révisé le 27 janyier 1988, accepté le 29 janvier 1988)

\begin{abstract}
Résumé. - La mobilité des dislocations dans le silicium monocristallin a été étudiée par mesures isothermes de frottement intérieur. Trois pics de relaxation ont été mis en évidence à des températures croissantes, leur existence étant liée aux conditions de déformation. Le premier pic a été attribué à la migration de décrochements géométriques avec une énergie $W_{\mathrm{M}}$ de $1,5 \mathrm{eV}$. Le second pic, d'énergie d'activation 1,95 eV, a été attribué à la création et à l'interaction des décrochements thermiques. Le dernier pic, d'énergie $2,4 \mathrm{eV}$, a été associé aux mouvements des décrochements thermiques dans le champ des atomes de dopant. L'énergie de formation des doubles décrochements déduite de ces essais $\left(F_{\mathrm{DK}}\right)$ est de $0,9 \mathrm{eV}$.
\end{abstract}

\begin{abstract}
Dislocation mobility in silicon single crystals has been studied by isothermal internal friction measurements. Three relaxation peaks have been observed at increasing temperatures, connected to the straining conditions. The first peak has been attributed to the migration of geometrical kinks, with activation energy $W_{\mathrm{M}}$ of $1.5 \mathrm{eV}$. The second one, with an activation energy of $1.95 \mathrm{eV}$, has been connected to the nucleation and the interaction of thermal kinks. The last one, with an activation energy of $2.4 \mathrm{eV}$, is associated with the motion of double kinds in the field of the doping atoms. Following this results, the double kink formation energy, $F_{\mathrm{DK}}$, has been found equal to $0.9 \mathrm{eV}$.
\end{abstract}

\section{Introduction.}

En raison de la hauteur des barrières de Peierls dans les matériaux covalents, la formation de doubles décrochements ne peut intervenir qu'à haute température. De plus, les expériences de plasticité ont montré l'existence d'une énergie de migration des décrochements importante $[1,2]$.

Les mécanismes de relaxation intrinsèques des dislocations ne peuvent donc être observés qu'à haute température et de fait, les essais sur le germanium n'ont révélé que l'existence d'un fond croissant avec la température aux températures supérieures à $0,5 T_{\mathrm{f}}\left(T_{\mathrm{f}}=\right.$ température absolue de fusion). Il convient de noter à ce propos que la gamme des fréquences de vibration disponible avec les méthodes classiques de mesure de frottement intérieur tend à repousser les effets de relaxation à des températures prohibitives.

Nous nous proposons donc d'utiliser des mesures isothermes de très basses fréquences, pour l'étude du silicium monocristallin déformé.

\section{Conditions expérimentales.}

Le matériau de base est du silicium FZ WASO dopé au bore et au phosphore. Des blocs $(20 \times 6 \times$ $6 \mathrm{~mm}^{3}$ ) orientés sont déformés à chaud par compression. Différentes déformations, répertoriées dans le tableau suivant, ont été effectuées.

Les échantillons de frottement intérieur ont été découpés suivant l'axe de compression et polis afin d'éliminer les sources superficielles de dislocations.

Le frottement intérieur est mesuré à l'aide d'un appareil précédemment décrit [6]. Il s'agit d'un pendule de torsion inversé de fréquence propre élevée, de l'ordre de $200 \mathrm{~Hz}$, soumis à des vibrations de torsion de fréquences comprises entre $10^{-4} \mathrm{~Hz}$ et $10 \mathrm{~Hz}$. Les parties réelles et imaginaires du module dynamique sont obtenues à partir de la réponse en phase et en amplitude de l'échantillon. Les amplitudes maximales de cisaillement sont de l'ordre de $10^{-5}$ et les mesures peuvent être faites sous un vide de $5 \times 10^{-6}$ Torr entre l'ambiante et, si la température de fusion de l'échantillon le permet, $2100 \mathrm{~K}$. 
Tableau I. - Conditions expérimentales des déformations par compression.

[Experimental conditions for compressive deformations.]

\begin{tabular}{|c|c|c|c|c|}
\hline $\begin{array}{c}\text { Déformation } \\
\mathrm{n}^{\circ}\end{array}$ & $\begin{array}{c}\text { Dopage } \\
\left(\text { at.cm }^{-3}\right)\end{array}$ & $\begin{array}{c}\text { Axe de } \\
\text { compression }\end{array}$ & $\begin{array}{c}\text { Déformation } \\
\text { totale (\%) }\end{array}$ & $\begin{array}{c}\text { Température } \\
(\mathrm{K})\end{array}$ \\
\hline 1 & Bore $9 \times 10^{16}$ & {$[1 \overline{1} 0]$} & 0,4 & 1320 \\
2 & Bore $9 \times 10^{16}$ & {$[1 \overline{1} 0]$} & 1,2 & 1050 \\
3 & Bore $9 \times 10^{16}$ & {$[1 \overline{1} 0]$} & 1,2 & $1150+820$ \\
4 & Phosphore $10^{13}$ & {$[2 \overline{3} 1]$} & 3,4 & 1220 \\
5 & Phosphore $6 \times 10^{18}$ & {$[2 \overline{3} 1]$} & 2,7 & 1220 \\
6 & Phosphore $6 \times 10^{18}$ & {$[2 \overline{3} 1]$} & 4 & 1220 \\
\hline
\end{tabular}

\section{Résultats expérimentaux.}

Aucun pic de relaxation de dislocations n'est observable aux températures inférieures à $1000 \mathrm{~K}$, auxquelles seul un fond exponentiel peut être observé (Fig. 1), confirmant les résultats antérieurs obtenus dans le silicium et le germanium [7]. Aux températures supérieures, des effets de relaxation supplémentaires, fonction des conditions de dopage et de déformation, se superposent au fond.

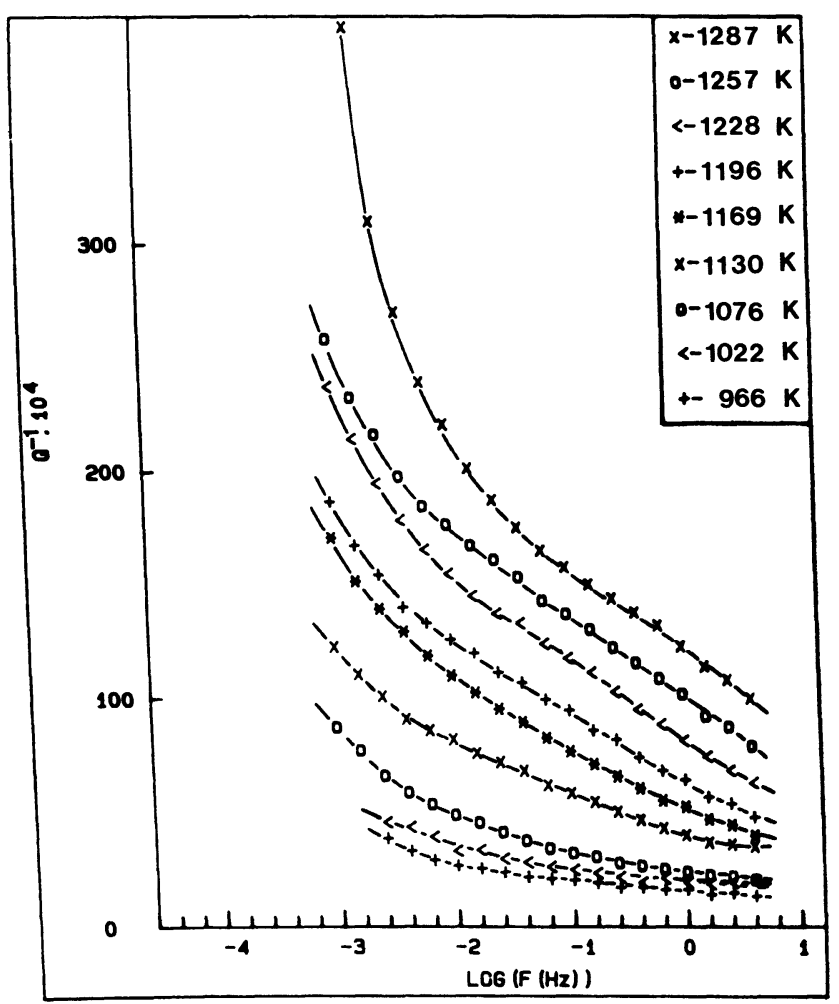

Fig. 1. - Spectres bruts de frottement intérieur isotherme. $\mathrm{Si}-\mathrm{B}$ (bore) déformé de $1,2 \%$ à $1150 \mathrm{~K}$ puis $820 \mathrm{~K}$.

[Isothermal internal friction spectra. Si-B (boron), $1.2 \%$ strained at $1150 \mathrm{~K}$ then $820 \mathrm{~K}$.]

L'analyse du fond, préalable à toute exploitation des pics de relaxation, doit être effectuée en plusieurs étapes.
Dans un premier temps, on constate (Fig. 2) que le fond est de la forme :

$$
\log \left(Q^{-1}-Q_{0}^{-1}\right)=\log A(T)-\alpha(T) \log \omega
$$

où $\omega$ est la pulsation d'excitation et $Q_{0}^{-1}$ le fond indépendant de la fréquence.

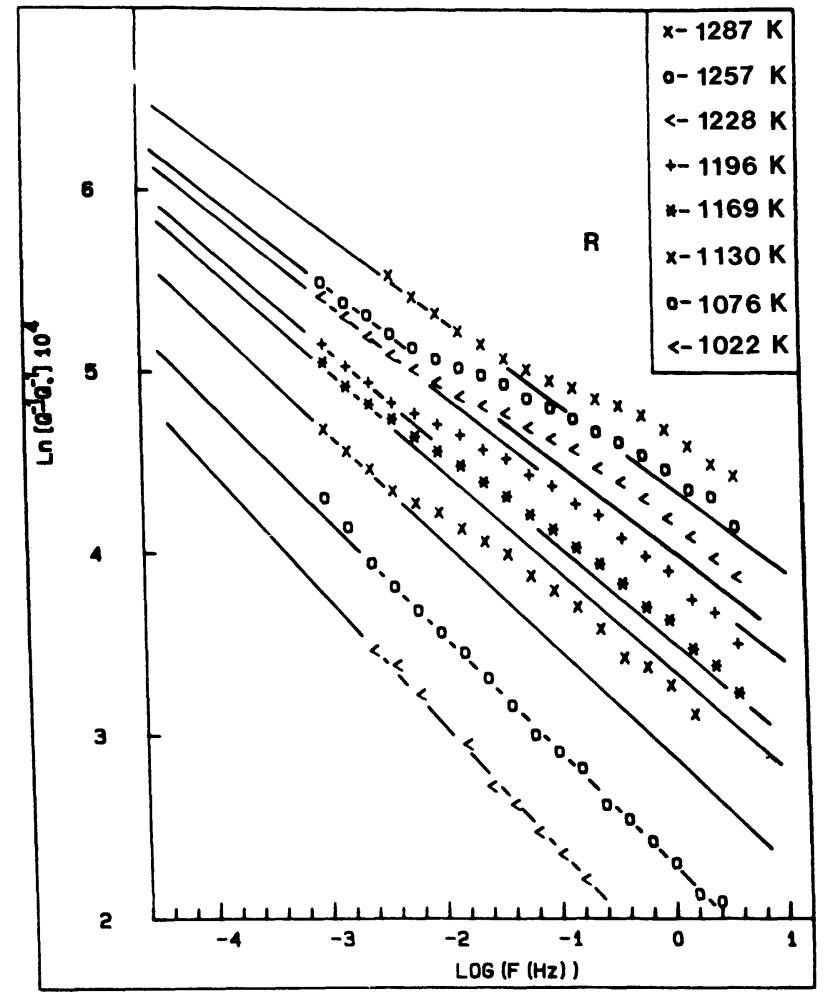

Fig. 2. $-\log \left(Q^{-1}-Q_{0}^{-1}\right)$ en fonction de $\log N$, d'après figure 1. Si-B, élimination du fond continu.

$\left[\log \left(Q^{-1}-Q_{0}^{-1}\right)\right.$ versus $\log N$, from figure 1. Removal of the low frequency background.]

La variation du coefficient $A(T)$ avec la température est donnée figure 3, pour $\omega=1$.

On obtient ainsi une énergie d'activation apparente $H_{\mathrm{A}}$ :

$$
\log A(T)=-\frac{H_{\mathrm{A}}}{k T} .
$$




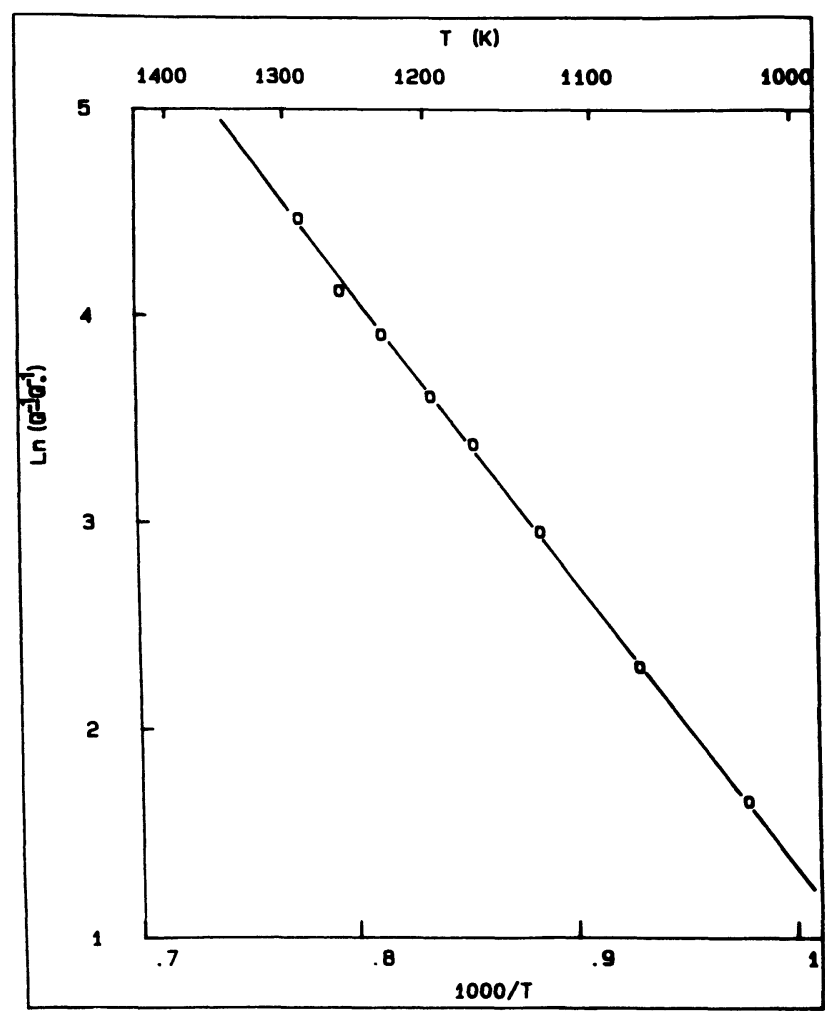

Fig. 3. - Variation du fond, en fonction de la température, pour $\omega=1$; Si-B.

[Background, as a function of temperature, for $\omega=1$.]

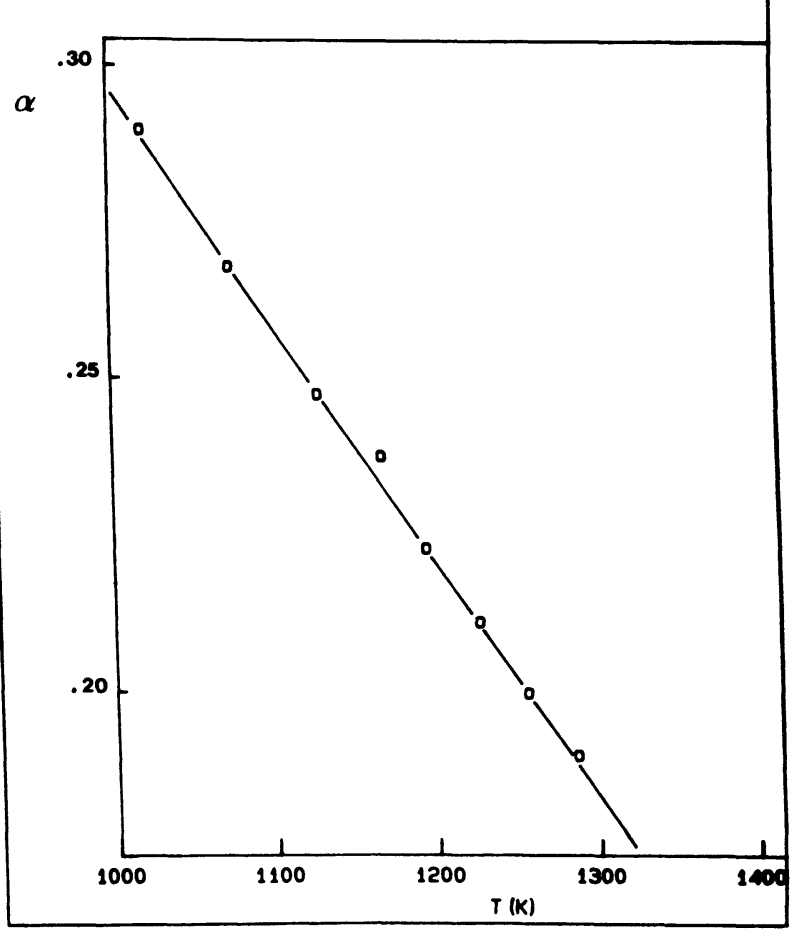

Fig. 4. $-\alpha(T)$ en fonction de la température.

[ $\alpha(T)$ versus temperature.]
Finalement la figure 4 montre les variations du paramètre $\alpha$ avec la température :

$$
\alpha(T)=-\beta T+\gamma \text {. }
$$

L'expression du fond est donc :

$$
Q_{\mathrm{F}}^{-1}-Q_{0}^{-1}=\frac{A_{0} \exp -\frac{H_{\mathrm{A}}}{k T}}{\omega^{-\beta T+\gamma}} \text {. }
$$

Le modèle de Schoeck [8] montre que l'énergie d'activation réelle du mécanisme associé à ce fond est :

$$
H_{\mathrm{F}}=\frac{H_{\mathrm{A}}}{\gamma} .
$$

Sur le tableau II, on peut constater que les paramètres $H_{\mathrm{F}}, \beta$ et $\gamma$ sont très sensibles à l'état microstructural de l'échantillon.

Tableau II. - Influence des conditions de déformation sur les paramètres $\beta, \gamma$ et $H_{\mathrm{F}}$.

[Influence of deformation conditions on the $\beta, \gamma$ and $H_{\text {F }}$ parameters.]

\begin{tabular}{|c|c|c|c|}
\hline $\begin{array}{c}\text { Déformation } \\
\text { (d'après le tableau I) }\end{array}$ & $\begin{array}{c}\beta \\
\left(10^{-3} \cdot \mathrm{K}^{-1}\right)\end{array}$ & $\gamma$ & $\begin{array}{c}H_{\mathrm{F}} \\
(\mathrm{eV})\end{array}$ \\
\hline 1 (dopage B) & 0,86 & 1,54 & 1,54 \\
2 (dopage B) & 0,26 & 0,53 & 1,74 \\
3 (dopage B) & 0,26 & 0,54 & 1,86 \\
4 (dopage P) & 0,70 & 1,11 & 1,93 \\
5 (dopage P) & 0,49 & 0,86 & 1,90 \\
6 (dopage P) & 0,34 & 0,77 & 1,86 \\
\hline
\end{tabular}

Dans le cas d'un dopage au Bore, l'énergie d'activation semble varier avec la température de déformation.

\section{Pics de relaxation.}

Après élimination du fond, les relaxations supplémentaires ne peuvent être caractérisées avec précision que dans le cas des échantillons dopés au bore, pour lesquels l'amplitude et la stabilité des pics sont suffisantes.

Trois pics, dont l'existence est liée à la température de déformation ont ainsi pu être isolés.

Le tableau III récapitule les conditions d'existence des pics, ainsi que les paramètres de relaxation, $\tau_{0}$ étant le temps de relaxation limite.

\section{Discussion.}

Les pics de relaxation ont été associés à des mécanismes intrinsèques de glissement des dislocations, glissement régi par la création et la migration des décrochements. Les décrochements sont abrupts, compte tenu de l'importance de la barrière de Peierls de $2^{\mathrm{e}}$ espèce [9]. Aux températures auxquel- 
Tableau III. - Conditions d'existence et paramètres de relaxation des pics de frottement intérieur.

[Conditions defining the existence and the relaxation parameters of the internal friction peaks.]

\begin{tabular}{|c|c|c|c|}
\hline Pic & $\begin{array}{c}\text { Condition } \\
\text { de déformation }\end{array}$ & $\begin{array}{c}H \\
(\mathrm{eV})\end{array}$ & $\begin{array}{c}\tau_{0} \\
(\mathrm{~s})\end{array}$ \\
\hline $\mathrm{P}_{1}$ & 1 & 2,36 & $1,3 \times 10^{-10}$ \\
& 2 & 2,39 & $0,8 \times 10^{-10}$ \\
$\mathrm{P}_{2}$ & 1 & 1,50 & $2 \times 10^{-8}$ \\
$\mathrm{P}_{3}$ & 3 & 1,95 & $1,3 \times 10^{-8}$ \\
\hline
\end{tabular}

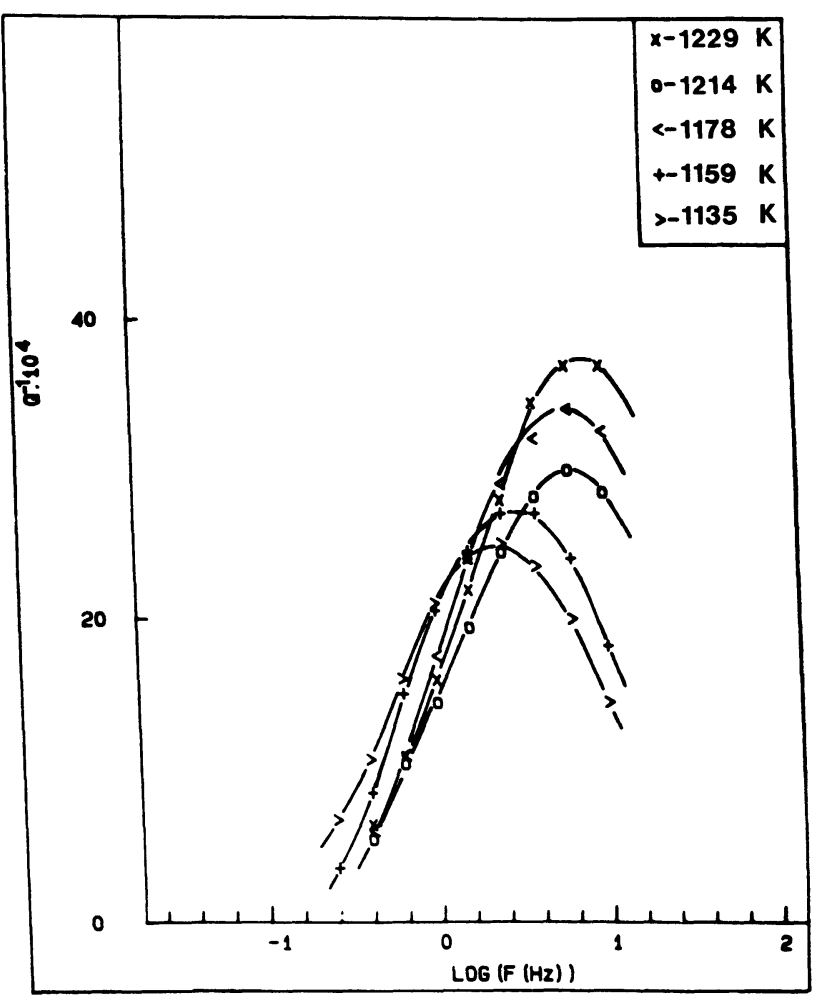

Fig. 5. - Pic $P_{1}$ obtenu après soustraction du fond, pour la déformation à $1320 \mathrm{~K}$.

$\left[P_{1}\right.$ peak, after low frequency background removal, in a specimen strained at $1320 \mathrm{~K}$.]

les $W_{\mathrm{M}}$ est inférieur à $k T$, la migration est thermiquement activée. Sous l'action de faibles contraintes, la vitesse d'un décrochement peut s'exprimer sous la forme :

$$
V_{\mathrm{k}}=\nu_{\mathrm{D}} \frac{a^{2} b h}{k T} \exp -\frac{W_{\mathrm{M}}}{k T}(\sigma-\varepsilon / \delta J)
$$

$\nu_{\mathrm{D}}:$ fréquence de Debye ;

$b$ : vecteur de Burgers ;

$h$ : distance entre vallées ;

$a$ : période le long de la ligne de dislocation;

$\delta J$ : défaut de complaisance ;

$\sigma$ : contrainte appliquée ;

$\varepsilon \quad$ : déformation.

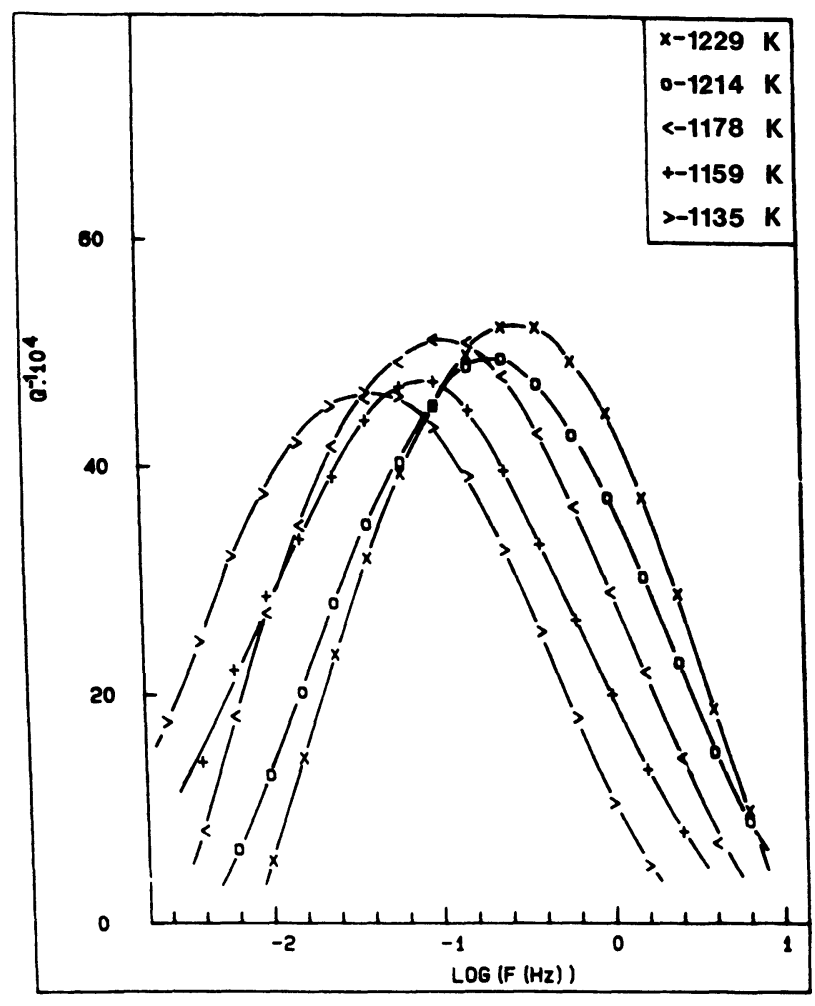

Fig. 6. - Pic obtenu après déformation à $1050 \mathrm{~K}$.

[Relation peak after straining at $1050 \mathrm{~K}$.]

Trois régimes de vitesse des dislocations peuvent alors être définis :

a) Pour les segments non parallèles aux vallées :

$$
v_{\mathrm{d}}=v_{\mathrm{k}} c_{\mathrm{k}} h \quad[9]
$$

$c_{\mathrm{k}}$ étant la densité linéaire de décrochements.

b) Pour les segments ancrés parallèles aux vallées, on peut distinguer, d'après le formalisme de Hirth et Lothe [10], deux régimes :

- Pour des segments de longueur moyenne supérieure à la longueur critique $\ell_{\mathrm{c}}$ :

$$
\ell_{\mathrm{c}}=\sqrt{2 a} \exp \left(\frac{F_{\mathrm{DK}}}{2 k T}\right)
$$

où $F_{\mathrm{DK}}$ est l'énergie de formation d'un double décrochement :

$$
v_{\mathrm{d}}=\frac{h}{a} v_{\mathrm{k}} \exp \left(-\frac{F_{\mathrm{DK}}}{2 k T}\right) .
$$

- Pour des segments courts, de longueur moyenne $L$ inférieure à $\ell_{\mathrm{c}}$ :

$$
v_{\mathrm{d}}=\frac{h L}{a^{2}} v_{\mathrm{k}} \exp \left(-\frac{F_{\mathrm{DK}}}{k T}\right) \text {. }
$$

Le pic $\mathrm{P}_{2}$ n'apparaissant qu'après déformation de haute température est associé au déplacement de 


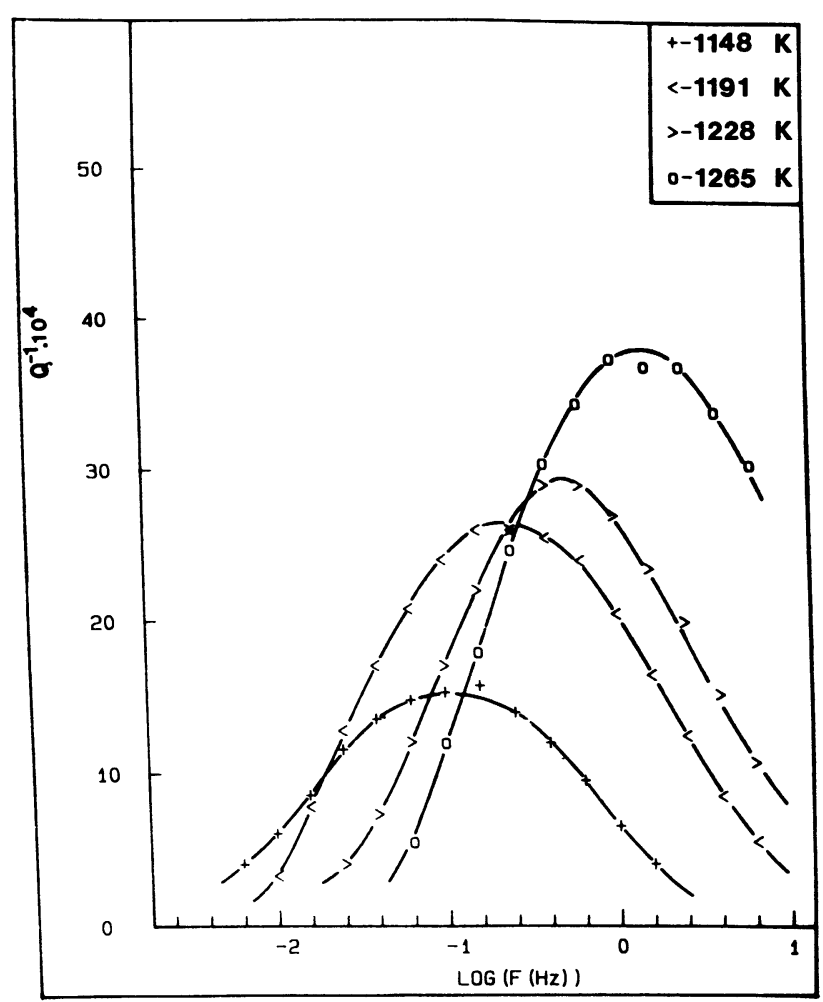

Fig. 7. - Pic $\mathrm{P}_{2}$ obtenu après déformation à $1320 \mathrm{~K}$.

$\left[\mathrm{P}_{2}\right.$ peak after straining at $1320 \mathrm{~K}$.]

segments désorientés. Les valeurs suivantes des paramètres de relaxation ont été obtenues :

$$
\begin{aligned}
E_{2} & =1,50 \mathrm{eV} \\
\tau_{02} & =\frac{\delta J k T}{\rho C_{\mathrm{k}} a^{2} b^{2} h^{2} \nu_{\mathrm{p}}}=2 \times 10^{-8} \mathrm{~s}
\end{aligned}
$$

où $\rho$ est la densité de dislocations. L'énergie d'activation $E_{2}(1,50 \mathrm{eV})$, correspond alors à l'énergie de migration des décrochements $W_{\mathrm{M}}$.

La valeur du temps de relaxation limite est tout à fait compatible avec les données expérimentales :

$$
\begin{aligned}
\rho & =4 \times 10^{7} \mathrm{~cm}^{-2} \\
\Delta j & =G \delta J=\frac{\rho p^{2}}{10}=6 \times 10^{-3}
\end{aligned}
$$

$G$ étant le module de cisaillement.

En prenant $C_{\mathrm{k}}=1 / 5 b$, ce qui correspond à des segments complètement désorientés et répartis de façon aléatoire dans le plan de glissement, on trouve un temps de relaxation limite de $10^{-8} \mathrm{~s}$ comparable à la valeur expérimentale $\left(2 \times 10^{-8} \mathrm{~s}\right)$.

Les pics $P_{1}$ et $P_{3}$ apparaissent après des déformations de plus basse température, ils sont donc associés au déplacement de segments parallèles aux vallées. Les énergies associées, supérieures à $W_{M}$, impliquent la création de doubles décrochements.

D'après le formalisme de Hirth et Lothe [10], les

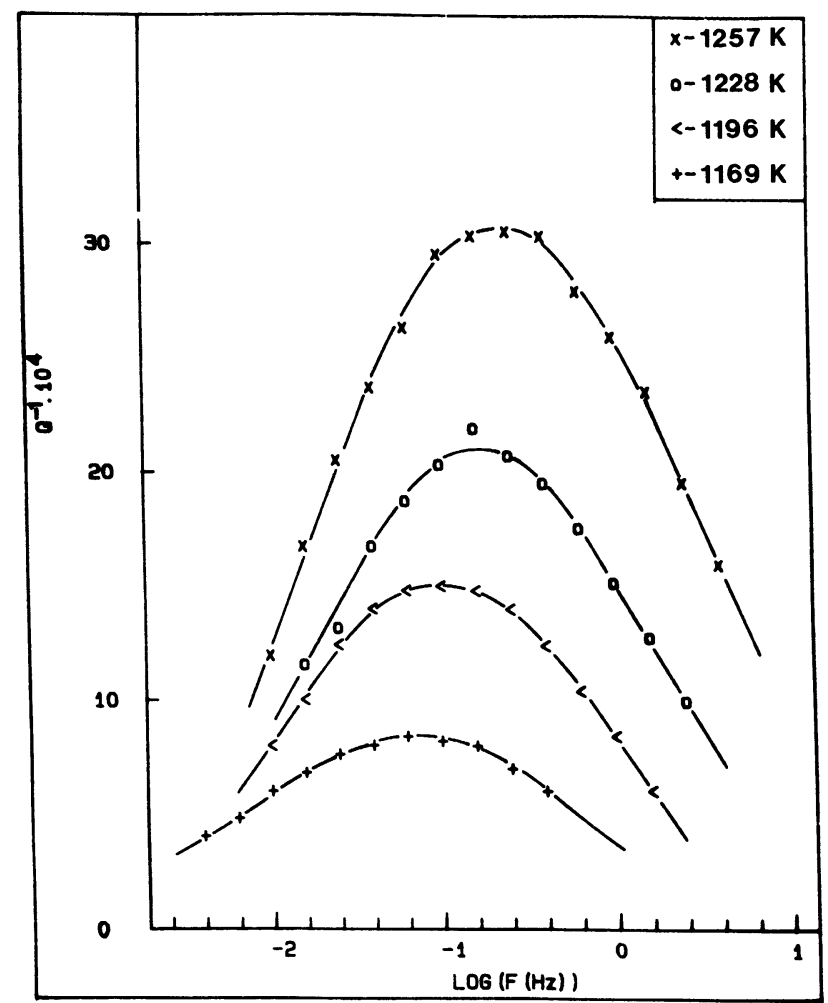

Fig. 8. - Pic $\mathrm{P}_{3}$ obtenu après double déformation à 1150 puis $820 \mathrm{~K}$.

$\left[\mathrm{P}_{3}\right.$ pead obtained after a double straining at 1150 then $820 \mathrm{~K}$.]

énergies $E_{1}$ et $E_{3}$, correspondant aux pics $\mathrm{P}_{1}$ et $\mathrm{P}_{3}$, sont de la forme :

$$
\begin{aligned}
& E_{1}=W_{\mathrm{M}}+F_{\mathrm{DK}} \\
& E_{2}=W_{\mathrm{M}}+\frac{1}{2} F_{\mathrm{DK}} .
\end{aligned}
$$

Compte tenu de la valeur précédemment trouvée pour $W_{M}$, on en déduit :

$$
F_{\mathrm{DK}}=0,9 \mathrm{eV} \text {. }
$$

valeur nettement inférieure à l'énergie de migration.

Ces valeurs sont à rapprocher de celles trouvées par Louchet, par mesures directes de la vitesse des dislocations [10].

Les temps de relaxation limite correspondants sont de la forme :

$$
\begin{aligned}
\tau_{01} & =\frac{\delta J k T}{\rho \nu_{\mathrm{D}} \rho b^{2} h^{2}} \\
\tau_{03} & =\frac{\delta J k T}{\rho \nu_{\mathrm{D}} a b^{2} h^{2}}
\end{aligned}
$$

$P_{1}$ étant relié aux segments courts $\left(\ell<\ell_{c}\right)$ et $\mathrm{P}_{3}$ aux segments longs.

Il convient cependant de noter que dans la gamme de température explorée $\ell_{\mathrm{c}}$ reste intérieure à 
$10^{-1} \mu \mathrm{m}$, ce qui pour des segments aussi courts conduirait à un pic de faible amplitude $\left(<10^{-4}\right)$, la valeur expérimentale étant 100 fois supérieure. Il y a donc une contradiction apparente entre le régime de vitesse qui correspond à des segments courts et l'intensité de relaxation imposant des longueurs de segments beaucoup plus importantes.

Pour lever cette difficulté, nous supposerons l'existence d'obstacles à la migration des crans sur les lignes de dislocation. Si la distance entre ces obstacles est inférieure à $\ell_{c}$, chaque portion, entre obstacles, de la ligne de dislocation obéit au régime [14].

$v_{\mathrm{s}}=J h \boldsymbol{\ell}, J$ étant le taux de nucléation des doubles décrochements.

Pour que la vitesse globale de la ligne de dislocation reste égale à $v_{\mathrm{s}}$, il suffit de supposer que les obstacles introduisent un temps d'attente pour les

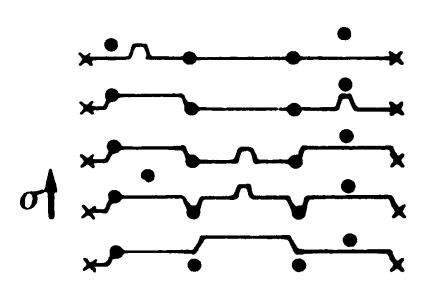

Fig. 9. - Franchissement des obstacles par les doubles décrochements.

[Obstacles overcoming by double kinks.] simples décrochements mais qu'ils sont franchis instantanément lorsque deux décrochements de signes opposés atteignent un obstacle. Ce mécanisme est illustré figure 9.

En ce qui concerne la nature de ces obstacles, l'hypothèse la plus réaliste est de les identifier aux atomes de bore qui sont effectivement en concentration suffisante $\left(9 \times 10^{6}\right.$ at. $\left.\mathrm{cm}^{-3}\right)$ pour vérifier la condition $\ell<\ell_{c}$ et suffisamment stables (sites substitutionnels) pour ne pouvoir être traînés par les dislocations. Ce mécanisme n'est $a$ priori envisageable qu'à faibles contraintes [12].

Le pic $\mathrm{P}_{1}$ est donc à rapprocher du mouvement des décrochements dans le champ des dopants.

La constante de temps $\tau_{01}$ associée au mécanisme proposé est alors $\left(\tau_{01}=(\delta J k T) /\left(\rho \nu_{\mathrm{D}} \ell b^{2} h^{2}\right)\right)$ en très bon accord avec la valeur expérimentale.

Quant au pic $\mathrm{P}_{3}$, dont l'énergie correspond au régime d'interaction entre décrochements, il ne peut exister qu'après une déformation à basse température, à laquelle les dislocations ont pu être arrachées de leur nuage de dopants.

Finalement, le fond continu, dont l'énergie varie de $W_{\mathrm{M}}$ à $W_{\mathrm{M}}+F_{\mathrm{DK}} / 2$ suivant la température de déformation, peut être associé au glissement non réversible des dislocations par absorption des décrochements par les nœuds étendus du réseau.

Selon les proportions de segments parallèles ou non aux vallées, l'énergie d'activation du fond évoluera entre $W_{\mathrm{M}}$ et $W_{\mathrm{M}}+1 / 2 F_{\mathrm{DK}}$.

\section{Bibliographie}

[1] Louchet, F., Inst. Phys. Conf. Serv. 60 (1981) 35.

[2] FARber, B. Ya, IUnin, S. et Nikitenko, V. T., Phys. Stat. Solidi A 97 (1986) 49.

[3] Geru, N. et Williams, D. J., J. Appl. Phys. 53 (1982) 3595.

[4] Welsch, G., Mitchell, T. E. et Gibala, R., Phys. Stat. Solidi A 15 (1973) 225.

[5] Moller, H. J. et JENDRICH, U., In deformation of Ceramic Materials II (118 Plenum Press, New York and London) 1984, p. 25.

[6] Woirgard, J., Sarrazin, Y. et Chaumet, H., Rev. Sci. Instrum. 48 (1977) 1322.
[7] Southgate, P. D. et Mendelson, K. S., J. Appl. Phys. 36 (1965) 2685.

[8] Schoeck, G., Bisogni, E. et Shyne, J., Acta Metall. 12 (1964) 1466.

[9] Brailsford, A. D., Phys. Rev. 122 (1961) 778.

[10] LOUCHET, F., Microscopy of Semiconducting Materials (1981) p. 35.

[11] HiRTH, J. P. et Lothe, J., In Theory of Dislocations (MeGraw Hill, New-York) 1968.

[12] HirSCH, P. B., Microscopy of semiconducting Materials (Oxford) 1983, p. 1. 